Nervenarzt 2008 · 79:703-705

DOI 10.1007/s00115-008-2457-7

Online publiziert: 4. April 2008

๑) Springer Medizin Verlag 2008

G. Brasse ${ }^{1} \cdot$ O. Stammel ${ }^{2} \cdot$ P. Siemens ${ }^{3} \cdot$ R. Töpper ${ }^{2}$

${ }^{1}$ Max-Planck-Institut für Kognitions- und Neurowissenschaften, Leipzig

${ }^{2}$ Abteilung für Neurologie, Asklepios-Klinik Harburg, Hamburg

${ }^{3}$ Abteilung für Radiologie, Asklepios-Klinik Harburg, Hamburg

\title{
Thrombose eines venösen Angioms mit sekundärem Stauungsinfarkt
}

\section{Fallbeschreibung}

\section{Anamnese}

Der 26-jährige, bis dato gesunde Patient stellte sich mit seit 2 Tagen bestehenden und in der Intensität zunehmenden Kopfschmerzen vor, welche als konstant, von nuchal nach frontal links ausstrahlend beschrieben wurden. Zusätzlich berichtete er wiederholtes schwallartiges Erbrechen ohne Übelkeit. Abgeschlagenheit bestünde bereits seit mehreren Wochen. Erwähnenswert ist ein fieberhafter Infekt 3 Wochen zuvor.

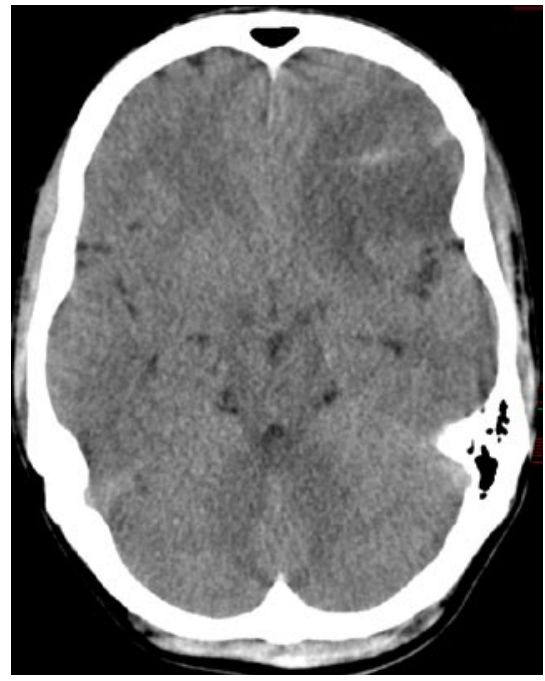

Abb. $1 \Delta$ CCT nativ 10.10.2006: hypodense Raumforderung im frontobasalen Marklager links, innerhalb der Raumforderung zeigt sich eine hyperdense tubuläre Struktur

\section{Klinischer Befund und paraklinische Befund}

Wir sahen einen 26-jährigen Patienten in leicht reduziertem Allgemeinzustand. Der allgemeinklinische Untersuchungsbefund war unauffällig, kein Fieber. Neurologisch bestand endgradige Nackensteifigkeit und ein bis auf linksseitige Ptosis regelhafter Hirnnervenbefund. Motorik, Sensibilität, Koordination, Stand und Gangprüfung blieben ohne Auffälligkeiten. Neuropsychologisch wurde fremdanamnestisch von Antriebsarmut und Verlangsamung in den vorangegangenen Tagen berich-

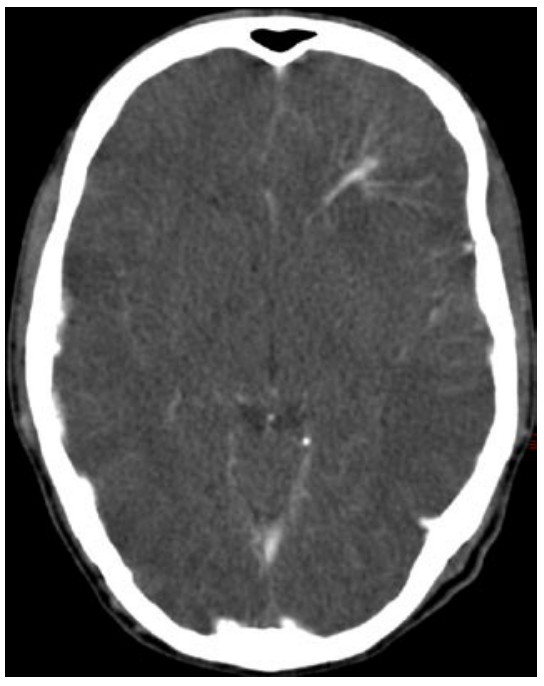

Abb. $2 \Delta$ In der venösen CT-Angiographie ist der Abbruch der drainierenden Vene als Ausdruck der Thrombose erkennbar tet, die Bedside-Testung erbrachte keine Hirnwerkzeugstörungen.

Pathologische Laborparameter zum Zeitpunkt der Aufnahme: CRP: $28 \mathrm{mg} / \mathrm{l}$ $(\mathrm{n}<5)$, ALAT: $175 \mathrm{U} / \mathrm{l}(\mathrm{n}=10-50)$, GGT: 75 $\mathrm{U} / \mathrm{l}(\mathrm{n}<60)$, D-Dimere $1,43 \mathrm{mg} / \mathrm{l}(\mathrm{n}<0,5)$. Leukozyten 10,2 /nl ( $n=3,5-9,8)$. Alle weiteren Routinelaborparameter lagen zum Zeitpunkt der Aufnahme im Normbereich. Im Verlauf: kein Nachweis pathologischer Parameter für Protein C und S, Faktor-VLeiden, APC-Resistenz. Lupus-Antikoagulans im Referenzbereich. Kein Nachweis einer Prothrombinmutation. Grenzwertige Erhöhung von AntikardiolipinAK IgM: 72 kU/l, im Verlauf 54 kU/l.

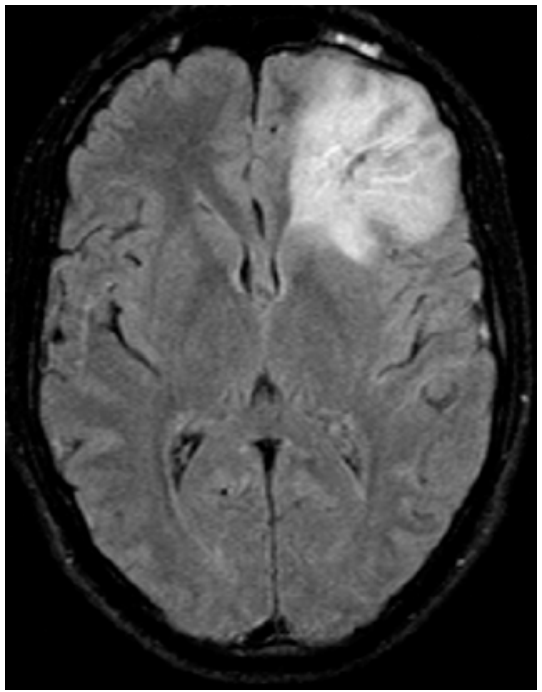

Abb. 3 A MRT 10.10.2006: (FLAIR ax) Solitäre Hirnvenenthrombose in einer transzerebalen Marklagervene links frontal mit ausgeprägtem Kongestionsödem 
Nervenarzt 2008 · 79:703-705

DOI 10.1007/s00115-008-2457-7

(c) Springer Medizin Verlag 2008

G. Brasse $\cdot$ O. Stammel · P. Siemens $\cdot$ R. Töpper

Thrombose eines venösen Angioms mit sekundärem Stauungsinfarkt

\section{Zusammenfassung}

Venöse Angiome ("developmental venous anomalies", DVA) gelten als embryonale Anlagebesonderheit des zerebralen Venensystems und stellen die häufigsten zerebralen Gefäßmalformationen dar. Ihr klinischer Stellenwert ist bis dato umstritten: Einzelfälle von Thrombosierung, venösen Infarkten und Blutungen im Zusammenhang mit venösen Angiomen wurden berichtet, im Wesentlichen gelten sie jedoch als gutartige Entität. Wir stellen den Fall eines 26-jährigen Patienten vor, bei dem das venöse Angiom durch eine Thrombosierung klinisch manifest wurde.

Schlüsselwörter

Venöse Malformation zerebral · Venöses Angiom · Venöser Infarkt · Zerebrale Gefäßmalformation · Hirnvenenthrombose

\section{Thrombosis of developmental venous anomaly and consecutive venous infarction}

\section{Summary}

Developmental venous anomalies (DVA) are congenital aberrations in the cerebral venous system reflecting failure of normal embryogenesis. They are considered the most common intracranial vascular malformation. Although they are generally regarded as benign entities, their clinical significance remains controversial - case reports do exist considering DVA to be the origin of thrombosis, cerebral ischemia, and hemorrhage. We report a 26-year-old patient suffering from thrombosis of a DVA.

\section{Keywords}

Developmental venous anomaly · Cerebral venous infarction - Intracranial vascular malformation · Intracerebral thrombosis

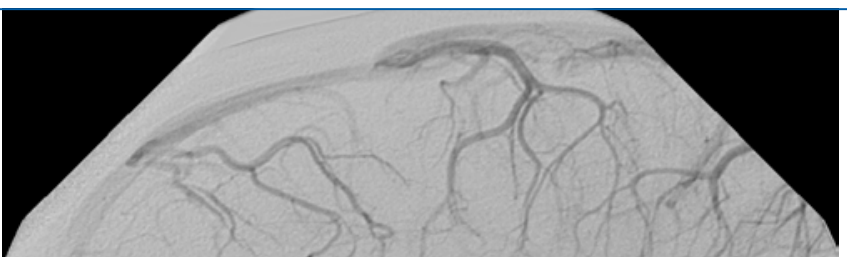

Abb. $4<$ Digitale Subtraktionsangiographie (DSA) der supraaortalen Gefäße vom 20.10.2006: selektive

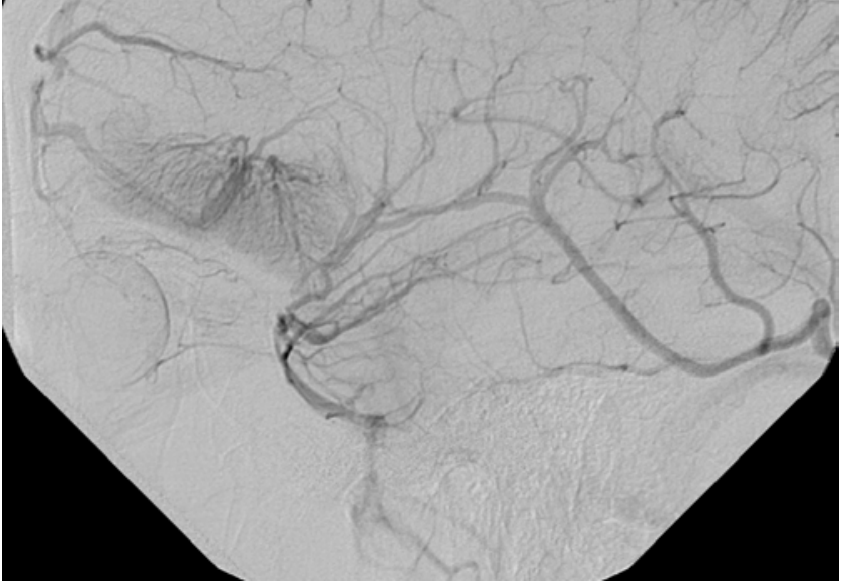
DSA der linken A. carotis interna, Spätphase des Phlebogramms: typische Gefäßmissbildung im Sinne eines venösen Angiomes. Die drainierende Vene zeigt eine zentrale Kontrastmittelaussparung als Ausdruck der stattfindenden Rekanalisierung und verläuft nach rostral zum vorderen Pol des Sinus sagittalis superior

\section{Diagnose, Therapie und Verlauf}

Nach Durchführung von CCT, CT-Angiographie und MRT (• Abb. 1, 2,3) konnte die Diagnose der Thrombosierung eines links frontal gelegenen venösen Angioms mit resultierendem Kongestionsödem gestellt werden.

Der Patient wurde systemisch PTT (partielle Thromboplastinzeit)-wirksam heparinisiert. Die Kopfschmerzen zeigten sich binnen $48 \mathrm{~h}$ rückläufig. Wir stellten die Behandlung auf ein fraktioniertes Heparinpräparat (Enoxaparin) s.c. in körpergewichtsadaptierer Dosierung um. Die Umstellung auf Phenprocuomon (International Normalized Ratio: 2-3) erfolgte im ambulanten Sektor.

Die ergänzend durchgeführte digitale Subtraktionsangiographie konnte eine High-flow-Komponete des Angioms ausschließen und damit den Befund einer venösen Anlagebesonderheit bestätigen (• Abb. 4).

Wir sahen den Patienten jeweils zum Zeitpunkt der Kontrollbildgebungen (• Abb.5). Eine anfänglich verminderte Belastbarkeit im Alltag bildete sich in den folgenden Wochen zurück. Die orale Antikoagulation wurde nach Ablauf von 3 Monaten beendet.

\section{Diskussion}

Die Einteilung der zerebralen Gefäßmalformationen geht auf McCormick [6] zu- rück. Im Wesentlichen werden heute unterschieden: venöses Angiom (DVA), kapilläres Angiom (Teleangiektasie), kavernöses Angiom (Kavernom), arteriovenöses Angiom (AV-Malformation), durale AV-Fistel.

Venöse Angiome stellen eine Persistenz embryologisch früher, "primitiver" venöser Drainagewege dar [1] und gelten als die häufigsten intrazerebralen Gefäßmalformationen [9, 10]. Ihre Inzidenz wurde in unterschiedlichen Übersichten mit 2,5\% (Post-mortem-Studie an 4069 Patienten [8]) bzw. 0,7\% (1,5 T-MRT bei 7266 Patienten einer Universitätsklinik [9]) angegeben.

Sie imponieren als radiär angeordnete Venen, die in ein zentral drainierendes, meist dilatiertes Gefäß münden („caput medusae“). Die Drainage erfolgt überwiegend über das oberflächliche zerebrale Venensystem. Ihr Stellenwert für den regulären venösen Abfluss des Parenchyms ist mittlerweile unbestritten [7]. Eine gehäufte Assoziation der venösen Angiome zu Kavernomen ist bekannt, ein möglicher gemeinsamer Pathomechanismus wird diskutiert [7]. Die klinische Relevanz der - bezüglich Lage und Größe stark variierenden - venösen Angiome bleibt Gegenstand von Diskussionen. Derzeit muss im Wesentlichen von einer klinisch asymptomatischen benignen Entität ausgegangen werden $[7,9,10]$, jedoch existieren Einzelfallberichte klinisch apparenter venöser Angiome: Hammoud 


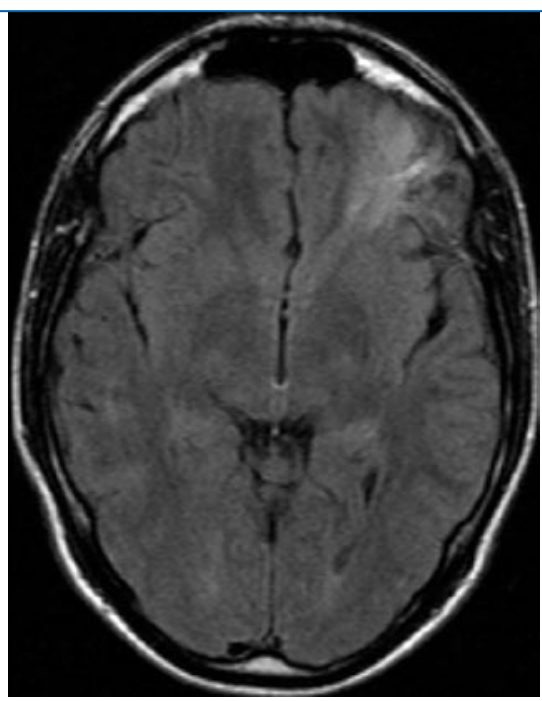

Abb. $5 \Delta$ Magnetresonanztomographie 06.11.2006 (FLAIR ax): im Vergleich zu den Voraufnahmen vom 10.10.2006 deutlich rückläufige Signalveränderungen links frontal mit kleiner wohl sekundär ischämischer Zone frontotemporal

et al. berichten von einer Thrombosierung mit venösem Infarkt [3], LovrencicHusjan et al. schildern eine Subarachnoidalblutung (SAB) im Zusammenhang mit einem thrombosierten venösen Angiom [5]. Vor diesem Hintergrund muss auch der Fall unseres Patienten als Besonderheit gewertet werden.

In Bezug auf die Entstehung der Thrombose weist die Kontrastierung des venösen Angioms in der späten venösen Phase des Angiogramms auf eine reduzierte Flussgeschwindigkeit in diesem Gefäßabschnitt hin. Über zusätzliche, prothrombotische Faktoren (in der Literatur beschriebene Besonderheiten bez. der Wandtextur, Stenosen an der Mündungsstelle, Relevanz des im Vorfeld stattgehabten Infektes) kann lediglich spekuliert werden.

\section{Fazit für die Praxis}

Die Optimierung bildgebender Verfahren sowie ihr vermehrter Einsatz wird die Anzahl nachgewiesener venöser Angiome in Zukunft möglicherweise ebenso erhöhen wie die ihrer pathologischen Veränderungen. Einem umfassenderen Verständnis dieser Entität und z. B. auch ihrer gehäuften Assoziation zu Kavernomen kann somit möglicherweise Vorschub geleistet werden. Bei häufig asymptomatischen Zufallsbefunden venöser Angiome bleibt die Bewertung im Zusammenhang mit dem klinischen Syndrom jedoch essenziell.

\section{Korrespondenzadresse}

\section{G. Brasse}

Max-Planck-Institut für Kognitions- und Neurowissenschaften

Stephanstraße 1 A, 04103 Leipzig

brasse@cbs.mpg.de

Interessenkonflikt. Der korrespondierende Autor gibt an, dass kein Interessenkonflikt besteht.

\section{Literatur}

1. Beall DP, Bell JP, Webb JR, Fish JR (2005) Developmental venous anomalies and cavernous angiomas: a review of the concurrence, imaging, and treatment of these Vascular Malformations. J Okla State Med Assoc 98: 11

2. Camacho DLA, Smith JK, Grimme JD et al. (2004) Atypical MR imaging perfusion in developmental venous anomalies. AJNR Am J Neuroradiol 25: 1549-1552

3. Hammoud D, Beauchamp N, Wityk R, Yousem D (2002) Ischemic complication of a cerebral developmental venous anomaly: case report and review of literature. J Comput Assist Tomogr 26: 633636

4. Huber G, Henkes H, Hermes M et al. (1996) Regional association of developmental venous anomalies with angiographically occult vascular malformations. Eur Radiol 6: 30-37

5. Lovrenncic-Huzjan A, Rumboldt Z, Marotti M, Demarin V (2004) Subarachnoidal haemorrhage headache from a developmental venous anomaly. Cephalalgia 24: 763-766

6. Mc Cormick WF (1966) The pathology of vascular (, arteriovenous") malformations. J Neurosurg 24: 807-816

7. Perrini P, Lanzino G (2006) The association of venous developemental anomalies and cavernous malformations: pathophysiological, diagnostic, and surgical considerations. Neurosurg Focus 21: E5

8. Sarwar M, Mc Cormick WF (1978) Intracerebral venous angioma. Arch Neurol 35: 323-325

9. Töpper R, Jürgens E, Reul J, Thron A (1999) Clinical significance of intracranial developmental venous anomalies. J Neurol Neurosurg Psychiatry 67: 234238

10. Truwit $C L$ (1992) Venous angiomas of the brain: history, significance and imaging findings. AJR Am J Roentgenol 159: 1299-1307

\section{Zelltod durch Tumor-Glutamat}

Wissenschaftler der Universität Erlangen-Nürnberg sind in der Erforschung von hirneigenen Tumoren einen entscheidenden Schritt vorangekommen: Sie identifizierten jetzt auf molekularer Ebene eine Ursache für Ödeme bei malignen Gliomen.

Wie die Forscher berichten, geben hirneigene Tumoren große Mengen des Neurotransmitters Glutamat ab. Dies führt zum Verlust von Nervenzellen im umliegenden Hirngewebe und reduziert die Überlebenszeit und die Lebensqualität des Patienten deutlich. Die Dysbalance an übermäßigem Glutamat ist darüber hinaus die Ursache des Hirnödems bei hirneigenen Tumoren. Durch solche Flüssigkeitsansammlungen im Gehirn kommt es unter anderem zu neurologischen Ausfällen.

Auf das Überleben des Patienten haben diese Untersuchungen zunächst keinen unmittelbaren Einfluss; jedoch schaffen die Ergebnisse besseren Einblick in die Biologie dieses Tumors. Bis heute haben die therapeutischen Konzepte der letzten 40 Jahre keine deutliche Verlängerung der Überlebenszeit oder gar Heilung gebracht. Das neue molekulare Verständnis eröffnet jedoch prinzipiell in den nächsten Jahren eine neue und eventuell effektivere therapeutische Option.

\section{Originalpublikation:}

Savaskan NE, Heckel A, Hahnen E et al. (2008) small interfering RNA-mediated $\mathrm{xCT}$ silencing in gliomas inhibits neurodegeneration and alleviates brain edema.

Nat Med. Epub

Quelle: Universität Erlangen www.uni-erlangen.de 